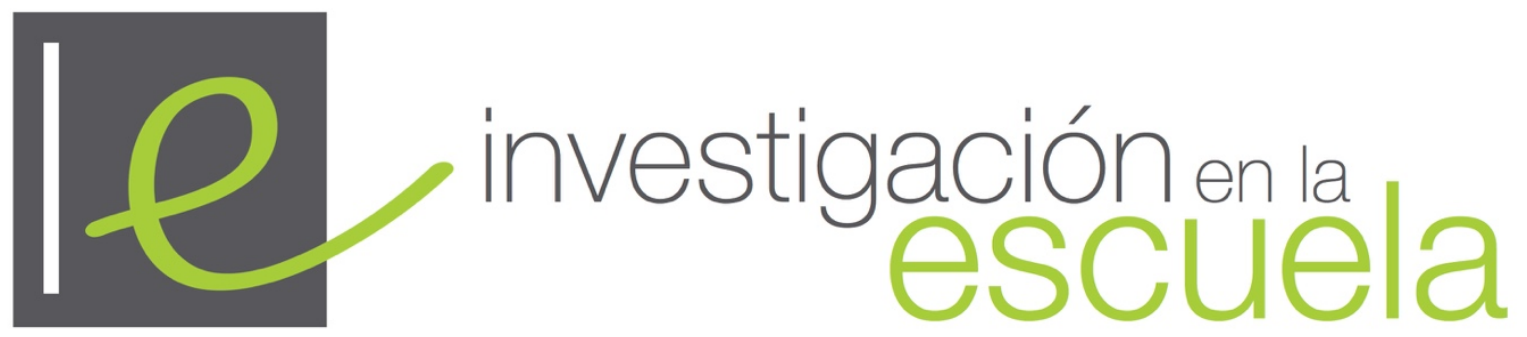

Revista internacional de investigación e innovación educativa

\title{
Prácticas Educativas en Educación rural: Enseñanza de las Ciencias Naturales
}

\author{
Adrián Galfrascoli', Maricel Lederbos', Silvia Veglia ${ }^{2}$ \\ Instituto Superior de Profesorado ${ }^{1}$, Escuela Normal Superior ${ }^{2}$ \\ Argentina
}

Citación: Galfrascoli, A., Lederhos, M. y Veglia, S. (2017). Prácticas Educativas en Educación rural: Enseñanza de las Ciencias Naturales. Investigación en la escuela, 93, 43-57. Recuperado de: http://www.investigacionenlaescuela.es/articulos/R93/R93-4

Resumen: Se expone y analiza el proceso de diseño e implementación de secuencias de actividades propuestas desde los enfoques de indagación y modelización para generar aprendizajes en Ciencias Naturales, en la escolaridad primaria en plurigrado rural. Se trata de los resultados parciales de un trabajo de investigación interinstitucional desarrollado en Argentina (Provincia de Santa Fe), por dos Institutos de Educación Superior, que tomó como objeto de estudio las prácticas de enseñanza de las Ciencias Naturales en dos escuelas rurales de Educación Primaria con grados agrupados. Se muestran criterios de diseño y análisis de secuencias para este contexto y se concluye que el enfoque de indagación-modelización promueve el desarrollo de habilidades de indagación y la construcción de modelos explicativos por parte de los estudiantes, en un escenario que enriquece este tipo de propuestas, el plurigrado.

Palabras clave: "Enseñanza primaria"; "escuela rural; "educación científica; "actividades escolares".

Educational Practices in Rural Education: Teaching Natural Sciences

Abstract: The process of designing sequences of activities to generate learning in the context of rural plurigrado is exposed and analyzed. This is the partial results of an interinstitutional research 
project developed in Argentina (Province of Santa Fe), by two Institutes of Higher Education, which took as object of study the teaching practices of the Natural Sciences in two rural schools of Primary Education with grouped degrees. Design criterions and sequences analysis are shown for this context and it is concluded that the investigation/inquiry-modelling/representation approach promotes the development of inquiry/investigation abilities and the construction of explanatory models by students, in a scene that enriches/improves this kind of proposals, the multigrade. Keywords: "Primary education"; "rural school"; "science education"; "school activities".

\section{Pratiques éducatives en education rurale: Enseigner les sciences naturelles}

Résumé: On expose et on analyse le processus de conception et d'application de séquences des activités proposées à partir des objectifs de la recherche et de la modélisation afin de produire des apprentissages en Sciences naturelles, à l'école primaire (en zone) rurale, regroupant plusieurs niveaux. Ce sont les résultats partiels d'un travail d'enquête inter-institutionnel, développé en Argentine (Province de Santa Fe), par deux Instituts d'Enseignement supérieur, qui a eu pour sujet d'étude les pratiques d'enseignement des Sciences naturelles dans deux écoles primaires rurales comprenant des niveaux regroupés. Les critères de conception et d'analyse des séquences pour ce cas sont mis en évidence. Ils conduisent à la conclusion que la mise en oeuvre d'un modèle opérationnel favorise le développement des compétences pour la recherche méthodique et initie l'élaboration de modèles explicatifs pour une partie des étudiants, dans une démarche enrichissant ce type d'initiative, le regroupement des scolaires de niveaux différents.

Mostsclés: "Enseignement primaire"; "école rurale"; "éducation scientifique"; "activités scolaires".

\section{Introducción}

Uno de los grandes desafíos de fines del siglo XIX a nivel mundial fue la alfabetización de grandes masas de pobladores (Graff, 1989). Un siglo después, el desafío que afrontan los países es alcanzar la alfabetización científica. Esto implica generar situaciones de enseñanza y de aprendizaje de las ciencias para que los niños y jóvenes adquieran los saberes necesarios para comprender los fenómenos naturales con los modelos explicativos de la ciencia escolar, para entender la Ciencia como una empresa humana, de construcción histórica y social (Ministerio de Educación, 2014). Para el desarrollo de la alfabetización científica son reconocidos como enfoques básicos: la modelización, la indagación y la contextualización de la enseñanza de las ciencias (Caamaño, 2011). Sin embargo, se detecta un vacío en la socialización de iniciativas combinando estas perspectivas para el aprendizaje de las Ciencias Naturales (CN), en plurigrado rural.

En la Argentina, los relevamientos oficiales (INFD, 2009) muestran la elevada presencia de las escuelas rurales (ER) en el total nacional y estudios anteriores muestran la complejidad que asume la labor docente en este tipo de instituciones (Gallegos, Calderón y García, 2009; Veglia, Galfrascoli y Lederhos, en prensa). El conocimiento pedagógico disponible para analizar la enseñanza en estos contextos y apoyar los procesos de construcción metodológica (Edelstein, 1996/1999) adecuados para el plurigrado, es escaso e inespecífico. Existe un desajuste entre los requerimientos didácticos y el conocimiento disponible y legitimado por los circuitos de formación que responde a la escuela monogrado. Este desajuste constituye para algunos especialistas una seria preocupación (Terigi, 2008a). Por otro lado, se sabe que la escuela rural posee potencialidades pedagógicas, humanas y relacionales aunque, según Feu i Gelis (2004) no fueron explicadas por lo que hay que profundizar su conocimiento (Oviedo, 2011).

En este marco, este trabajo pretende socializar el análisis del diseño e implementación de una secuencia que responde a la combinación indagación-modelización para la enseñanza de las $\mathrm{CN}$ en el 
contexto del plurigrado rural. Las preguntas que guiaron el estudio fueron: ¿Qué elementos y dimensiones debe contemplar el diseño e implementación de una secuencia de enseñanza de las $\mathrm{CN}$ para el contexto de plurigrado rural? ¿Qué aprendizajes de CN promueve la implementación de una secuencia de enseñanza basada en el modelo didáctico por indagación- modelización en un plurigrado? ¿Qué saberes pueden desarrollar los niños de ER caracterizados por su heterogeneidad cuando se les brinda la oportunidad de desarrollar procesos de indagación y modelización en el aula?

El estudio adoptó una naturaleza cualitativa, de alcance descriptivo (Gómez, 2009). Se pretendió avanzar en la descripción y explicación de la relación entre las variables del problema de investigación (la enseñanza por indagación-modelización y el aprendizaje de las $\mathrm{CN}$ ) mediante el diseño e implementación de una unidad didáctica de corte investigativo (De las Heras y Jiménez, 2011).

Dada la naturaleza del objeto de estudio, se optó por realizar un estudio de carácter de indagación intensivo antes que extensivo; el diseño implicó una triangulación metodológica de fuentes de información y el mismo método en dos situaciones diferentes (Pievi, 2009). La etapa de diseño implicó el análisis de los documentos curriculares oficiales y orientaciones sobre el tema. Luego, la información sobre la implementación de la secuencia de enseñanza y el aprendizaje de los estudiantes fue recolectada principalmente mediante la observación participante de las clases de CN. Esta información se complementó con el análisis de cuadernos de clase. Para el análisis de la enseñanza, la información de las observaciones se puso en diálogo con entrevistas a los docentes para profundizar sobre las dimensiones de la investigación que no fueron evidentes en la práctica.

\section{Acerca del contexto de investigación: el plurigrado rural}

El aula plurigrado o multigrado presenta diferencias significativas en comparación con el aula monogrado o de sección única. "Cuando pensamos en una clase, generalmente nos imaginamos al docente en el frente, ante su escritorio o paseando entre los bancos; y a todos los alumnos, escuchando una exposición o realizando alguna tarea, todos con la misma consigna o con el mismo ejercicio. La imagen del aula como la de un espacio en el que todos tienen más o menos la misma edad y hacen las mismas cosas..." (Anijovich y Mora, 2010, p. 101). Así se caracteriza la escuela urbana: una distribución de los cuerpos en el espacio del aula con una disposición misal (Diker, 2005). Se trata de la representación de la gramática escolar (Tyack y Cuban, 2001) más difundida, que llegó a ser legitimada durante la modernidad (Terigi, 2008b).

Las escuelas de educación primaria que forman parte del sistema educativo argentino, particularmente en la provincia donde se realizó el estudio, si bien presentan rasgos comunes, tienen diferencias significativas con relación a la cantidad de estudiantes matriculados, a la del personal docente asignado y al tipo de prácticas que las condiciones materiales posibilitan. Esas diferencias son más notorias entre establecimientos primarios urbanos y escuelas primarias rurales, que adoptan el plurigrado o multigrado como formato escolar (Aguerrondo, 2015).

En la Provincia de Santa Fe existen varios tipos de instituciones rurales con plurigrado. En este estudio centramos la atención en dos de ellos: los Centros Educativos Radiales (CER) son escuelas muy pequeñas que poseen solo un docente que tiene a cargo todos los grados, con una matrícula que no suele superar los 15 alumnos y depende administrativamente de otra escuela; y las escuelas de $4^{a}$ categoría, instituciones educativas que pueden ser polidocentes o unidocentes con una matrícula de hasta 60 alumnos. En ellas, uno de los docentes, además del dictado de clases, tiene a cargo la dirección del establecimiento.

Diferentes trabajos muestran las peculiaridades del aula plurigrado (Aguerrondo, (Coord.), 2015; Galfrascoli, 2013; INFD, 2009; Lederhos, Galfrascoli y Veglia, 2014; Terigi, 2008a). En una 
descripción sintética, se trata de una configuración didáctica (Litwin, 2000) específica de la ruralidad, una configuración pedagógica y didáctica multidimensional (Boix, 1995) caracterizada por una clase heterogénea, constituida por alumnos de diferentes grados que trabajan simultáneamente con un solo docente, compartiendo espacios y recursos en torno a una propuesta de aprendizajes, abierta a las variables del medio natural y social que se aprovechan como recursos. Respecto de esto último, se llegó a afirmar que la escuela rural tiene como soporte al medio social y natural (Abós Olivares, 2007).

Las dos escuelas del estudio son de cuarta categoría con personal único. Una de ellas, de la zona norte de la provincia, un docente/director está a cargo de un grupo de alumnos de seis grados (segundo a séptimo), de tres ciclos, que no supera los 20 estudiantes. La otra, ubicada en la zona centro-oeste de Santa Fe, también es de cuarta categoría, pero, como la cantidad de estudiantes se aproxima a los 30, funciona en doble turno, primer ciclo por la mañana y segundo ciclo, más séptimo grado, por la tarde.

\section{Los enfoques de indagación y de modelización para la enseñanza y el aprendizaje de las Ciencias Naturales en Plurigrado}

La enseñanza de la ciencia por o basada en la indagación se propuso en varios países con la intención de combatir la desmotivación o falta de interés de los estudiantes hacia la ciencia (Caamaño, 2011; Caamaño, 2012; Harlen, 2010; Sbarbati, 2015). Se trata de una metodología impulsada por Dewey hacia 1910 (Postigo y Greca, 2014) que surge como reacción frente al modelo de enseñanza tradicional pero que se distancia del aprendizaje por descubrimiento espontáneo (Furman, 2012). Se afirma que es una propuesta coherente con una visión de ciencia renovada y con teorías constructivistas de enseñanza y de aprendizaje.

Este enfoque de la enseñanza de las ciencias (Caamaño, 2012), “implica que los estudiantes trabajen de un modo similar al de los científicos, desarrollando comprensión desde la obtención y uso de evidencia para someter a prueba distintas formas de explicar los fenómenos que están estudiando" (Harlen, 2010, p. 3). Así, "usando las destrezas empleadas por los científicos para hacerse preguntas, obtener datos, razonar y revisar evidencias a la a luz de lo conocido, los estudiantes van sacando conclusiones, discutiendo resultados y construyendo nuevos conocimientos" (Sbarbati, 2015, p. 18). Furman (2012) propone tres modelos para diseñar las secuencias: el de Karplus, el de Kolb y el de Koch y Barton.

Por otro lado, respecto del término modelo, como señalan Gilbert, Boulter y Elmer (2000) (citados en Justi, 2011), "una definición bastante aceptada es aquella que considera que un modelo es la representación parcial de un objeto, un evento, un proceso o una idea, creado con un objetivo específico" (p. 86). Schwartz y otros (2009) (citados por Martí, 2012) definen al modelo como "una representación abstracta y simplificada de un sistema o fenómeno que hace explícitos y visibles sus elementos centrales y que puede ser usado para generar explicaciones y predicciones" (p. 91). También se ha definido desde el realismo cognitivo como "una entidad abstracta que se comporta como afirma o dicta la teoría a través de sus enunciados" (Adúriz Bravo, Labarca y Lombardi, 2014, p. 42).

Pueden identificarse dos grandes corrientes que han aportado ideas interesantes sobre los modelos que tienen connotaciones para la enseñanza de las ciencias. Una surge de la confluencia de los aportes de la psicología cognitiva (representada por los aportes de Johnson-Laird sobre la existencia y evolución de los modelos mentales) y la didáctica de la ciencia (Moreira, Greca y Rodríguez, 2002). En la otra, confluirían los aportes de la nueva Filosofía de la Ciencia y la Didáctica de las Ciencias (Adúriz Bravo, Labarca y Lombardi, 2014). En ésta, los argumentos no se centran tanto en lo que ocurre "dentro" de la mente de los individuos sino más bien en lo que la historia y la filosofía de la ciencia nos aportaron respecto de la construcción y evolución de las grandes teorías que la ciencia ha elaborado para explicar los fenómenos naturales en el marco de las comunidades 
científicas. Situada en esta corriente, reconocemos la propuesta de Arcà, Guidoni y Mazzoli (1990) que, siguiendo a Wittgenstein, presentan un modelo sobre el funcionamiento dialéctico del sistema cognitivo entendiéndolo en una interacción constante entre pensamiento, lenguaje y acción. Si bien sendas corrientes comparten una posición constructivista del conocimiento, su principal diferencia sería la concepción sobre los procesos cognitivos.

Este trabajo intenta demostrar que la indagación en vínculo con la modelización, se convierten en una potente alternativa para propiciar el desarrollo de secuencias en las que se incluyan diversidad de situaciones de enseñanza vinculadas entre sí. En tanto se ofrece a los alumnos la posibilidad de observar, diseñar experiencias, formular hipótesis y preguntas, resolver situaciones problemáticas, conceptualizar y explicar con el fin de comprender los fenómenos y comunicar el saber.

\section{Metodología del estudio}

El estudio partió de la necesidad de generar conocimiento didáctico específico para la enseñanza de las $\mathrm{CN}$ con una propuesta que asegure un camino de alfabetización científica para el contexto de plurigrado rural. El objetivo estuvo dirigido al logro de una secuencia superadora de aquellas que constituyen una transpolación del currículo propio del formato de escuela graduada (Galfrascoli, 2013; Lederhos et al., 2014; Terigi 2008a).

El problema abordado fue: ¿Cuáles son las características de las secuencias de enseñanza y aprendizaje que favorecen la indagación y modelización en multigrado? ¿Qué tipo de actividades son las más adecuadas para favorecer las habilidades de indagación y la construcción de modelos explicativos escolares en un contexto de plurigrado rural? ¿Cómo secuenciarlas? ¿Qué características deben reunir las secuencias para que sean adecuadas en el multigrado?

El objetivo general que orientó el trabajo fue analizar la enseñanza y el aprendizaje de CN por indagación-modelización en la implementación de secuencias didácticas para el plurigrado en ER santafesinas. Se formularon, además, dos objetivos específicos: a) analizar el diseño de una secuencia didáctica de $\mathrm{CN}$ basada en la indagación-modelización para el plurigrado (situaciones de enseñanza de indagación, construcción y evolución de modelos científicos escolares, adecuación al plurigrado); y b) analizar la implementación de una secuencia didáctica basada en la indagación-modelización (adecuación al plurigrado, saberes a enseñar y aprender, incentivación, secuencias de actividades) en las clases de $\mathrm{CN}$.

Las unidades de análisis fueron docentes y estudiantes del plurigrado. Se analizó la experiencia en dos escuelas rurales de diferentes regiones de la provincia de Santa Fe, una del Departamento Las Colonias (en la zona Centro Oeste) y la otra del Departamento General Obligado (de la zona Noreste).

Las dimensiones de análisis fueron: las características de la secuencia de enseñanzaaprendizaje que responde al modelo indagación-modelización; las características que la hacen adecuada (o no) al nivel primario y a la modalidad rural; y los logros de aprendizaje de los estudiantes sobre las $\mathrm{CN}$ en relación con la secuencia implementada. De modo que los resultados de la investigación fueron una descripción densa de la práctica de enseñanza de la secuencia de $\mathrm{CN}$ y su relación con los logros de aprendizaje, especificando características y posibles causas de los procesos planteados para CN, en Educación Primaria en contexto de plurigrado.

Para alcanzar los objetivos, el trabajo se desarrolló en dos fases. En la primera, se procedió a elaborar una secuencia de enseñanza de las $\mathrm{CN}$ que favoreciera la indagación y la construcción de modelos por parte de los estudiantes de un grupo multigrado rural desde una perspectiva constructivista. Y en la segunda, se implementó dicha secuencia y se evaluó su adecuación tanto para 
la modalidad rural, como para favorecer el desarrollo de habilidades indagatorias y la construcción de modelos explicativos escolares.

Las técnicas de recolección de datos que se aplicaron fueron:

- Análisis de documentos: se analizaron documentos curriculares nacionales: Núcleos de Aprendizajes Prioritarios, y los provinciales: Diseños Curriculares Jurisdiccionales, uno que organiza el Primer Ciclo de escuelas primarias, constituido por primero, segundo y tercer grado; el del Segundo Ciclo, que comprende cuarto, quinto y sexto grado, y el del Tercer Ciclo, del cual tomamos sólo el de séptimo grado. Este análisis permitió identificar y seleccionar un grupo de contenidos que se articularía en una propuesta de progresión de complejidad creciente. A partir de dicha selección y secuenciación, se elaboró una propuesta de actividades que tomó como tópico los materiales de construcción (que emplean los animales y, entre ellos, las personas). Para realizar dicho diseño se tuvieron en cuenta una serie de indicadores de las dimensiones importantes para este estudio: estrategias de indagación; estrategias de modelización; adecuación al plurigrado; concordancia con los contenidos a desarrollar; potencial motivador y coherencia interna. Luego, con dichos indicadores, se diseñó una tabla con la que se analizó la secuencia de actividades propuesta.

Tabla 1

Dimensiones de análisis e indicadores

\begin{tabular}{|c|c|c|}
\hline & $\begin{array}{l}\text { Dimensiones de } \\
\text { análisis }\end{array}$ & Indicadores \\
\hline $\begin{array}{l}\text { Objetivo } \\
\text { específico } 1\end{array}$ & $\begin{array}{l}\text { Concreción del } \\
\text { principio de la } \\
\text { indagación en la } \\
\text { secuencia. }\end{array}$ & $\begin{array}{l}\text { a. Se brindan oportunidades para formular } \\
\text { preguntas. } \\
\text { b. El docente propone algunas preguntas para } \\
\text { indagar. } \\
\text { a. Se permite que los estudiantes propongan una } \\
\text { forma experimental para responder la pregunta } \\
\text { de indagación. } \\
\text { b. El docente propone realizar uno o varios } \\
\text { experimentos. } \\
\text { a. Se presentan situaciones en las que los alumnos } \\
\text { buscan nueva información que les permita } \\
\text { terminar de resolver el problema o ampliar lo } \\
\text { que han aprendido. } \\
\text { b. El docente brinda información que explica y } \\
\text { organiza lo aprendido. } \\
\text { a. Se ofrece ayuda a los alumnos para organizar lo } \\
\text { aprendido. } \\
\text { b. Se ayuda a sistematizar la información que surgió } \\
\text { de las actividades anteriores y a construir } \\
\text { nuevos conceptos. } \\
\text { Los estudiantes pueden expresar (externalizar) } \\
\text { modelos intuitivos. }\end{array}$ \\
\hline
\end{tabular}




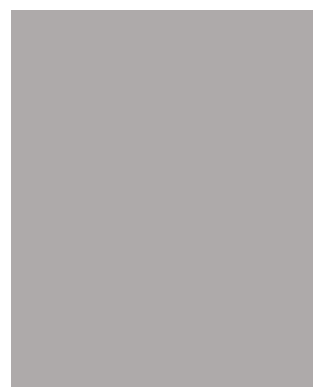

Objetivos

específicos 1

y 2

Adecuación al plurigrado.

Saberes a enseñar y aprender (contenidoshabilidades).

Incentivación

(implicanción para los aprendizajes)

Tipos y relaciones entre actividades de la/s secuencia/s.
Los estudiantes pueden contrastar modelos propios con modelos de pares.

Los estudiantes pueden contrastar modelos propios con los modelos propuestos por la ciencia escolar. Los estudiantes pueden contrastar sus propios modelos en diferentes estados de avance.

Los estudiantes pueden aplicar modelos para resolver problemas de la vida cotidiana.

Presencia de actividades de resolución individual. Presencia de actividades de resolución en pequeños grupos (inter-nivel). Presencia de actividades en pequeños grupos por grado.

Presencia de actividades en grupo clase.

Uso del entorno para identificar problemas, obtener información, reflexionar, imaginar, recolectar muestras.

Participación de actores sociales de la comunidad.

Se posibilita el aprendizaje significativo de nuevos conceptos.

Comprensión de los saberes.

Desarrollo de habilidades cognitivas y manipulativas (procedimientos de diferentes niveles de complejidad, se incluyen las habilidades lingüísticas). Expresión de actitudes de cooperación, reflexión crítica, apertura y diálogo respetuoso.

Los estudiantes no se ven interpelados por la propuesta.

Los estudiantes demuestran interés en algunos momentos.

Los estudiantes mantienen interés de manera sostenida.

Las actividades presentan articulación lineal en torno a la temática de trabajo por año.

Las actividades son adecuadas a los diferentes objetivos de la/s clase/s (inicio, desarrollo y cierre).

Presencia de actividades nodales de interacción/articulación (llamaremos así a las actividades que requieren de la participación de estudiantes de diferentes grados).

- Entrevistas: se entrevistó a dos docentes (uno por institución investigada). Se aplicó un cuestionario estructurado en base a 18 preguntas que pretendían indagar sobre cuatro dimensiones: datos de la trayectoria del docente, características institucionales, modalidades de organización del aula y relación escuela-comunidad. Esto posibilitó conocer las decisiones 
didácticas de los docentes, sus fundamentos, los modos de agrupamientos que propiciaban y el manejo de las $\mathrm{CN}$ como saber a enseñar y aprender.

- Observación participante: un miembro del grupo de investigación estuvo a cargo del desarrollo de cuatro clases, una por semana, de una duración de tres horas y cinco minutos cada una (lo que equivale a un día de clases completo en el medio rural según Decreto $\mathrm{N}^{\mathrm{o}}$ 4720/61). El tiempo se distribuyó en dos módulos de duración variable (el uso del tiempo respondió a la capacidad de atención y la necesidad de descanso de los estudiantes, acordado con ellos en contrato pedagógico ad hoc) con un intervalo de unos 15 minutos. La docente a cargo del grado estuvo presente durante todo el tiempo de la experiencia como oyente y observadora con participación, sus impresiones fueron recuperadas en la entrevista informal que se desarrolló al finalizar cada clase. Asimismo, otros miembros del equipo registraron la actividad por medio de notas, registro fotográfico y audiovisual. La observación posibilitó registrar el proceso de implementación de la propuesta en un diálogo constante con los actores.

\section{Enseñar y aprender Ciencias Naturales mediante la indagación-modelización en plurigrado rural. Análisis de los resultados}

A partir de lo observado en las clases y las entrevistas, pudo evaluarse la propuesta. La secuencia acercó a los niños a la forma de hacer ciencia que tienen los científicos. Esta red de actividades se sustentó en una trama que dio respuestas a la complejidad del trabajo en plurigrado y habilitó situaciones para plantear preguntas y buscar soluciones empleando modelos propios (del/los niño/os) y prestados (por los pares o por la ciencia escolar a través del docente).

Las actividades propiciaron el trabajo colaborativo entre estudiantes de diferentes niveles de experiencia y/o conocimiento. En ellas, los niños más avanzados pudieron ejercer el rol de monitores, y a su vez, para desempeñarse en ese papel tuvieron que afianzar o mejorar sus propios saberes. En ese intercambio entre niños de diferentes niveles se pusieron en diálogo, compararon, contrastaron, se transformaron, se construyeron o se consolidaron diferentes modelos explicativos para abordar los fenómenos en estudio.
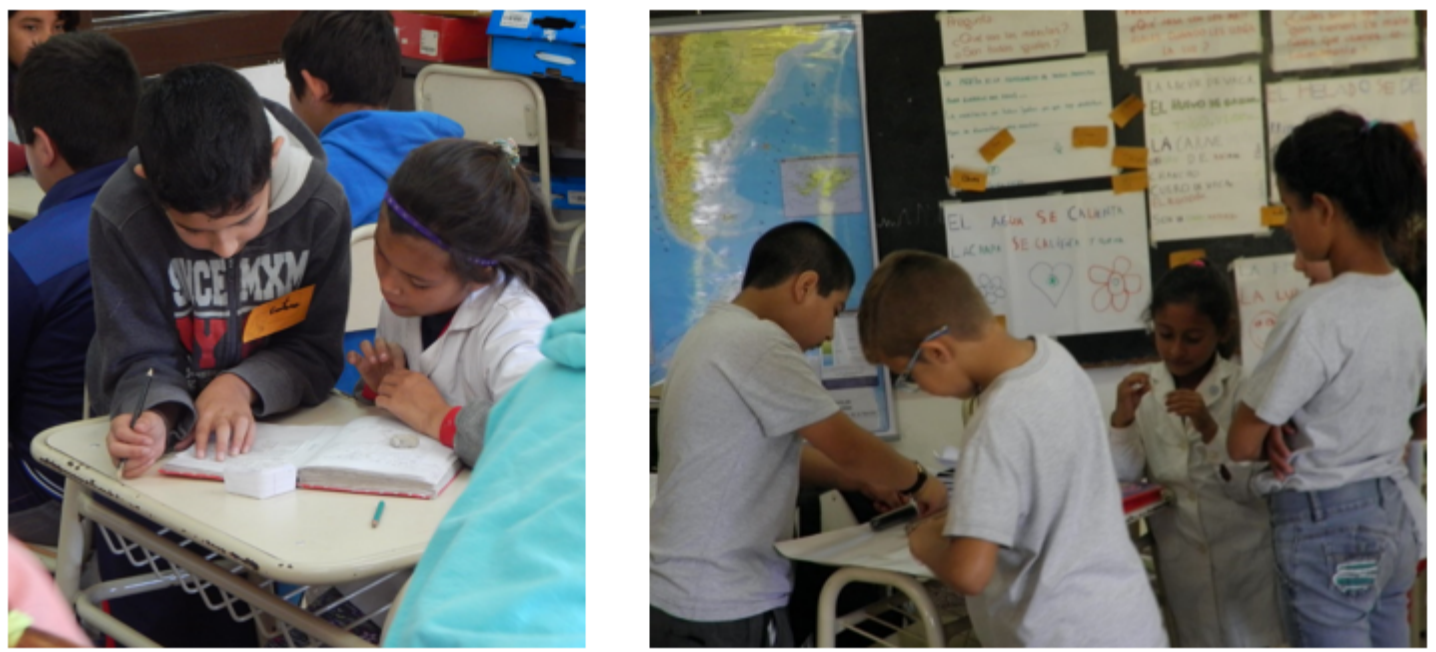

Figura 1. Desarrollo de actividades desarrolladas por niños de diferentes grados 
Se constató que una de las variables de gran influencia al momento de implementar propuestas de enseñanza de las $\mathrm{CN}$ es el tipo de agrupamientos de estudiantes. De ello depende la selección de contenidos, el tipo de actividades, organización de la tarea, etc. Se identificó que el intercambio es más enriquecedor cuando el grupo es heterogéneo. La interacción lleva a los alumnos más grandes a ayudar y aportar ideas más elaboradas a los pequeños, mientras que éstos, demandan a los compañeros de más edad explicaciones que los obligan a reflexionar sobre sus modelos y a mejorarlos.

En el marco de una indagación guiada, juega un papel importante la identificación y formulación de preguntas de investigación. En la implementación de la secuencia se ha podido observar que identificar y formular preguntas investigables son tareas que requieren una atención didáctica especial por las dificultades que les plantea a los estudiantes del Nivel Primario.

Los niños del ciclo superior manifestaron dificultades para formular preguntas. Corroboramos que a "preguntar y preguntarse" se aprende, la formulación de interrogantes es un procedimiento que ha de desarrollarse en escenarios que planteen intencionalmente su construcción.

Los niños del primer ciclo, en cambio, tienen mucha curiosidad, se hacen y formulan preguntas, pero no todas ellas son preguntas investigables. En estas situaciones es conveniente considerar que "para ser fructífera, la guía del docente debe involucrar un diagnóstico continuo de qué están pensando y comprendiendo los alumnos” (Furman, 2012, p. 26).

A partir de la actividad disparadora común se conformaron grupos heterogéneos diversos, algunos formularon preguntas de investigación sencillas con cierta autonomía y poca guía del docente; otros grupos, en los que los estudiantes no pudieron generar preguntas, requirieron ayuda de docente, quien les asignó una pregunta de investigación; y grupos en los que las preguntas formuladas eran confusas o no posibilitaban iniciar la búsqueda de pruebas empíricas para poder contrastar las hipótesis.

La pregunta que guiaría la investigación surgió del consenso del grupo clase; quedó registrada a la vista durante todo el proceso de indagación con el fin de no perder el rumbo de búsqueda. Se pudo observar cómo los niños pueden generar acuerdos, en un clima democrático y participativo, elaborando argumentos y tratando de disuadir al resto de aceptar una idea, algo que caracteriza el quehacer de los científicos.

En clase no hemos empleado el término hipótesis. Transformamos a un lenguaje más coloquial este término para facilitar la comunicación y resignamos -para más adelante en el tiempouna aproximación más precisa de la noción. Ante las preguntas: “¿A ustedes qué les parece si...? ¿Qué sucedería si...? ¿Cómo podemos hacer para...?”, los niños respondieron con gran creatividad, formularon hipótesis sin saber que lo estaban haciendo. Se registraron todas las respuestas dadas y se decidió colectivamente quiénes pondrían a prueba las seleccionadas mediante el diseño de experiencias.

El trabajo con experiencias en el marco de las secuencias tuvo diferentes niveles de complejidad. En algunos casos se proponía que los estudiantes inventaran la forma de buscar información por medio de un experimento con gran libertad; en otros, se sugería el protocolo a seguir por medio de una guía. En ambos tipos de actividades la indagación actuó como principio rector.

$\mathrm{Al}$ introducir este enfoque en la secuencia se identificaron diversas reacciones: los niños pequeños se mostraron muy entusiasmados, fue significativa la atención que pusieron y el intercambio de ideas que generó una propuesta que los llevó a pensar sobre la interacción de la luz con los materiales. Asimismo, los niños de los grados superiores mostraron gran interés en la formulación de anticipaciones, en la preparación de mezclas y en la contrastación de sus modelos iniciales con los datos que aportaba la experiencia. 
Todas estas actividades se encuadran en la fase de exploración (Uzcátegui y Betancourt, 2013). La intervención estuvo ajustada a las demandas de aprendizaje, al nivel de complejidad de la tarea y a la necesidad de orientar las discusiones hacia el objeto de reflexión favoreciendo la expresión y la argumentación haciendo que la palabra no sea monopolizada por los más grandes.

En la etapa de reflexión aparece la necesidad de comparar los resultados obtenidos con las predicciones elaboradas. Con respecto a la inquietud de obtener nueva información para reestructurar sus ideas, se propusieron textos, pero el vocabulario específico se introdujo cuando los alumnos realizaron experiencias, no se presentó terminología nueva a priori de la experiencia y de la problematización de ideas. Notamos que esta decisión fue importante para favorecer la expresión de los alumnos y explicitar sus modelos que luego adoptarían un "nombre" y crecerían en complejidad, a medida que se desarrollara la secuencia.

En la comunicación de las ideas, tanto la observación como el análisis de los cuadernos de clase muestran que a los niños les cuesta más expresarse por escrito que empleando dibujos. Los esquemas que realizan frecuentemente son incompletos, y hay que orientarlos con preguntas para que los puedan mejorar. En los grupos superiores es más frecuente encontrar producciones en las que se combinan esquemas con textos.

Se pudieron observar condiciones adecuadas para que los niños modelicen articulando el proceso de elaboración de modelos con la intervención experimental sobre el fenómeno a estudiar. En el caso de los alumnos del primer ciclo, manipularon los distintos materiales, los compararon, experimentaron el comportamiento frente a la luz, anticiparon las formas de las sombras, las dibujaron, produjeron sombras y contrastaron los resultados con sus supuestos expresados gráficamente. Los alumnos de segundo ciclo mezclaron diferentes materiales, predijeron el comportamiento de algunos de ellos al mezclarlos con agua, o al calentarlos, esquematizaron, escribieron, inventaron categorías conceptuales, buscaron las que propone la ciencia escolar en los textos, se evaluaron, etc. Notamos que la complejidad de las representaciones elaboradas aumenta a medida que nos alejamos de primer grado.

En síntesis, la propuesta implementada consistió en un conjunto de situaciones de enseñanza y de aprendizaje que permitieron a docentes y estudiantes desarrollar habilidades conducentes a un mejor conocimiento de la realidad, mediante estrategias para la construcción del conocimiento científico en el marco de la enseñanza de las ciencias basada en la indagación y la modelización.

\section{Conclusiones}

La enseñanza y el aprendizaje por indagación-modelización constituye una potente propuesta para el aprendizaje de las $\mathrm{CN}$ que se enriquece al desarrollarse en plurigrado rural. A esta conclusión llegamos a partir del diseño e implementación de una secuencia didáctica sobre el eje "los materiales y sus cambios" que combinó ambos enfoques.

El diseño de la secuencia afrontó dos desafíos: atender a las particularidades de los grados agrupados y a la modalidad e intencionalidad pedagógica de la indagación y la modelización. Esto obligó a continuos ajustes en esta etapa del proyecto. La dificultad mayor estuvo centrada en diseñar actividades para niños de plurigrado a lo largo de la secuencia, que reconocieran los objetivos de aprendizaje específicos de cada edad, considerando los documentos curriculares obligatorios, diagramados para el aula graduada. En la implementación de la secuencia, también hubo factores externos que desafiaron su curso, principalmente por el ausentismo.

La secuencia consideró un conjunto de situaciones que permitieron a los alumnos desarrollar habilidades que los pusieron en mejores condiciones para pensar, hacer y hablar sobre el mundo, de manera más crítica. Mediante estrategias amplias y variadas, los estudiantes pudieron compartir 
instancias de diálogo, reflexión y discusión fundada en las que tuvieron oportunidad de poner a prueba algunos de los modelos explicativos que habían construido, revisarlos, ampliarlos, modificarlos o conservarlos.

De acuerdo con Caamaño (2011, 2012), Harlen (2010) y Sbarbati (2015), constatamos que las actividades propias del modelo de indagación motivan a los alumnos y favorecen potentes modos de pensar, actuar y hablar en ciencias. También identificamos que los postulados de Martí (2012) sobre la potencialidad de la modelización para la representación mental, explicación y predicción aparecen en las diferentes edades. Todos los estudiantes lograron representaciones que utilizaron para explicar y predecir fenómenos. Aunque es preciso mencionar que las actividades de modelización son eficaces cuando se realiza un buen diagnóstico del grupo y se ajusta su complejidad a las potencialidades de los sujetos, facilitando el empleo de múltiples formas de representación: gráficas, verbales y simbólicas.

Finalmente, consideramos que el trabajo resulta un aporte al conocimiento didáctico específico para la enseñanza de las $\mathrm{CN}$, y brinda una propuesta que asegura un camino de alfabetización científica para el contexto de plurigrado rural.

\section{Referencias}

Abós Olivares, P. (2007). La escuela rural y sus condiciones ¿̇tiene implicaciones en la formación del profesorado? Aula Abierta, 35 (1, 2), 83-90.

Adúriz Bravo, A., Labarca, M. y Lombardi, O. (2014). Una noción de modelo útil para la formación del profesorado de química. En C., Merino, M., Arellano Johnson y A., Adúriz Bravo (Edits.). Avances en Didáctica de la Química: Modelos y lenguajes (pp. 37-50). Recuperado de: http://www.ccpems.exactas.uba.ar/biblio/Modelos\%20y\%20Lenguajes.pdf

Aguerrondo, I. (Coord.) (2015). Las escuelas del medio rural. Buenos Aires: Talleres Gráficos Manchita.

Arcà, M., Guidoni, P. y Mazzoli, P. (1990). Enseñar ciencia. Cómo empezar: reflexiones para una educación científica de base. España: Paidós Educador.

Caamaño, A. (2011). Enseñar química mediante la contextualización, la indagación y la modelización. Alambique, Didáctica de las Ciencias Experimentales, (69), 21-34.

Caamaño, A. (2012). Cómo introducir la indagación en el aula. Alambique Didáctica de las Ciencias Experimentales, (70), 83-91.

De las Heras, M.A. y Jiménez, R. (2011). La enseñanza del ser vivo en primaria a través de una secuencia de estrategias indagatorias. Alambique Didáctica de las Ciencias Experimentales, (67), 71 78.

Diker, G. (2005). Los sentidos del cambio en educación. En G., Frigerio y G., Diker. (Comps.) Educar: ese acto político (pp. 127-137). Buenos Aires: Del Estante.

Edelstein, G. (1996/1999). Un capítulo pendiente: El método en el debate didáctico contemporáneo. En A., Camilloni, M.C., Davini, G. Edelstein, E., Litwin, M., Souto y S., Barco. Corrientes didácticas contemporáneas ( $3^{\mathrm{a}}$ reimpresión). Buenos Aires: Paidós.

Feu i Gelis, J. (2004). La escuela rural en España: apuntes sobre las potencialidades pedagógicas, relacionales y humanas de la misma. Revista Digital eRural, Educación, cultura y desarrollo rural, 2 (3), 1-13.

Galfrascoli, A. (2013). Reflexiones acerca de las peculiaridades de la enseñanza de las ciencias naturales en grados agrupados de escuelas rurales. Revista Aula Universitaria, 15, 31-42.

Gallegos, L., Calderón, E. y García Rivera, B. (2009). El uso de materiales didácticos para la enseñanza de las ciencias en la escuela multigrado. En X Congreso Nacional de Investigación 
Educativa. Veracruz, Veracruz. Recuperado de:

http://www.comie.org.mx/congreso/memoriaelectronica/v10/pdf/area_tematica_05/pone ncias/1141-F.pdf

Gómez, M.M. (2009). Introducción a la metodología de la investigación científica. Córdoba: Brujas.

Graff, H.J. (1989). El legado de la alfabetización: constantes y contradicciones en la sociedad y la cultura occidentales. Revista de Educación, (288), 7-34.

Harlen, W. (2010). Principios y grandes ideas de la educación en ciencias. Hatfield: Association for Science Education. Recuperado de: www.innovec.org.mx

INFD (2009). Serie recomendaciones para la elaboración de diseños curriculares. Educación Rural, Buenos Aires: Ministerio de Educación. Recuperado de: http://cedoc.infd.edu.ar/upload/rural.pdf

Justi, R. (2011). Las concepciones de "modelo" de los alumnos, la construcción de modelos y el aprendizaje de las ciencias. Una relación compleja y centran en la enseñanza de las ciencias. En A., Caamaño. (Coord.). Didáctica de la física y la química, (pp. 85-103). Barcelona: Graó.

Lederhos, M., Galfrascoli, A. y Veglia, S. (2014) ¿Qué y cómo enseñan Ciencias Naturales en plurigrado los docentes de escuelas rurales santafesinas?. Krínein Revista de Educación, (11), 125-144.

Litwin, E. (2000). Las configuraciones didácticas. Buenos Aires: Paidós.

Martí, J. (2012). Aprender ciencias en la educación primaria. Barcelona: Graó.

Ministerio de Educación, Ciencia y Tecnología (2004). Seminario "Educación de la Población Rural en América Latina: Alimentación y educación para todos". Santiago de Chile, 3, 4 y 5 de agosto de 2004. Disponible en: http://www.me.gov.ar/curriform/publica/edrural.pdf

Moreira, M., Greca, I. y Rodríguez, L. (2002). Modelos mentales y modelos conceptuales en la enseñanza y aprendizaje de las ciencias. Revista Brasileira de Pesquisa em Educaşao em Ciências, 2 (3), 36-56.

Oviedo, T. (2011). Consultoría sobre la modalidad plurigrado como alternativa para las comunidades rurales. CIIE - Centro de Investigación e Innovación Educativa - MEC. Recuperado de: http://www.mec.gov.py/cms_v2/adjuntos/2401

Pievi, N. (2009). Documento metodológico orientador para la investigación educativa. Buenos Aires: Ministerio de educación de la Nación.

Postigo, D. y Greca, I. (2014). Uso de la metodología de la indagación para la enseñanza de nociones sobre fuerzas en primer ciclo de la escuela primaria. Revista Enseñanza de la Física, 26 (número extra), 265-273.

Sbarbati, N. (2015). Educación en ciencias basada en la indagación. Revista CTS, 28 (10), 11-22.

Terigi, F. (2008a). Organización de la enseñanza en los plurigrados de las escuelas rurales. (Tesis de maestría).

Terigi, F. (2008b). La invención del hacer: La enseñanza en los plurigrado de escuelas rurales en Argentina. En VII Seminario Redestrado. Buenos Aires, Argentina.

Tyack, D. y Cuban, L. (2001). En busca de la utopia. Un siglo de reformas de las escuelas pública. México: Fondo de Cultura Económica.

Uscátegui, Y. y Betancourt, C. (2013). La metodología indagatoria en la enseñanza de las ciencias: una revisión de su creciente implementación a nivel de Educación Básica y Media. Revista de Investigación, 78 (37), 109-127.

Veglia, S., Galfrascoli, A. y Lederhos, M. (en prensa). Desafíos del plurigrado rural santafesino: decidir qué y cómo enseñar Ciencias Naturales. Ministerio de Educación, INFD. 


\section{Información sobre los autores}

Autor: Adrián Galfrascoli

Institución: Profesor del Instituto Superior de Profesorado No 4, Santa Fe, Argentina

Email: adriang@trcnet.com.ar

Autor: Maricel Lederhos

Institución: Profesora de la Escuela Normal Superior No 30, Santa Fe, Argentina

Email: maricellederhos@gmail.com

Autor: Silvia Veglia

Institución: Profesora de la Escuela Normal Superior No 30, Santa Fe, Argentina

Email: vazzolerveglia@gmail.com 


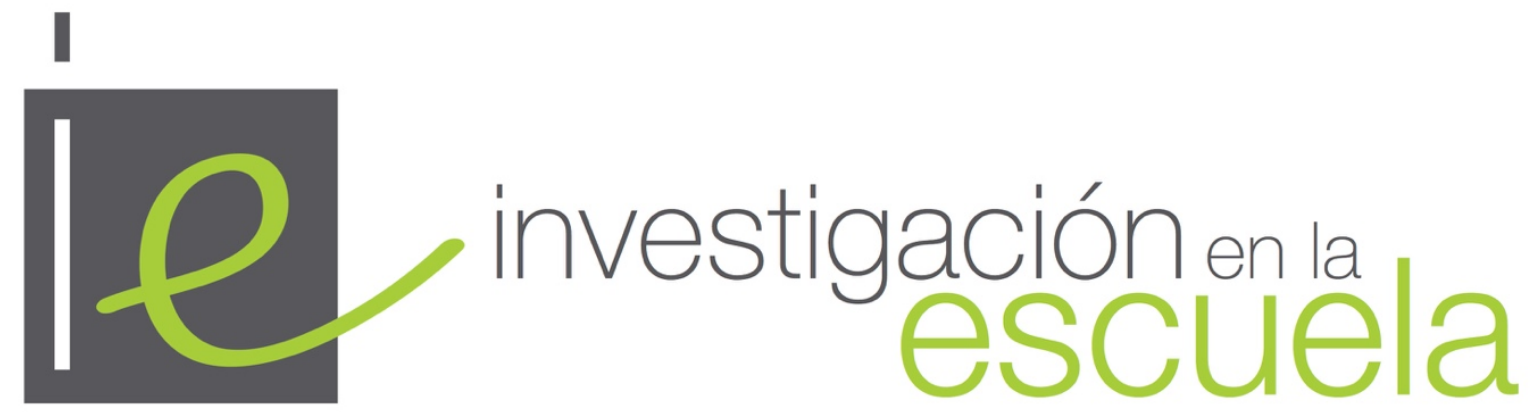

Revista académica evaluada por pares y de acceso abierto

Número 93

28 de diciembre de 2017

ISSN 2443-9991

\section{(c)}

SOMERIIGHISRESERVEDLOS/as lectores/as pueden copiar, mostrar, y distribuir este artículo, siempre y cuando se de crédito y atribución al autor/es y a Investigación en la Escuela, se distribuya con propósitos nocomerciales, no se altere o transforme el trabajo original. Más detalles de la licencia de CreativeCommons se encuentran en http://creativecommons.org/licenses/by-nc-sa/3.0 Cualquier otro uso debe ser aprobado en conjunto por el autor/es, o Investigación en la Escuela.

ư

Revista Editada por la Universidad de Sevilla. https://editorial.us.es/es/revistainvestigacion-en-la-escuela

Contribuya con comentarios y sugerencias en la web de la revista. Por errores y sugerencias contacte a secretaria@investigacionenlaescuela.es 


\section{Investigación en la escuela}

Consejo de dirección: Ana Rivero García (Universidad de Sevilla), Nicolás de Alba Fernández (Universidad de Sevilla), Pedro Cañal de León (Universidad de Sevilla), Francisco F. García Pérez (Universidad de Sevilla), Gabriel Travé González (Universidad de Huelva), Francisco F. Pozuelos Estrada (Universidad de Huelva)

Dirección: Ana Rivero García y Nicolás de Alba Fernández

Secretaría de edición: Elisa Navarro Medina

\section{Consejo editorial}

José Félix Angulo Rasco. Universidad de Cádiz Rosa Ma Ávila Ruiz. Universidad de Sevilla Pilar AzcárateGoded. Universidad de Cádiz Juan Bautista Martínez Rodríguez. Universidad de Granada

Nieves Blanco García. Universidad de Málaga Fernando Barragán Medero. Universidad de La Laguna

José Carrillo Yáñez. Universidad de Huelva José Contreras Domingo. Universidad de Barcelona. Luis C. Contreras González. Universidad de Huelva Ana $\mathbf{M}^{\mathbf{a}}$ Criado García-Legaz. Universidad de Sevilla Rosario Cubero Pérez. Universidad de Sevilla José $\mathbf{M}^{\mathbf{a}}$ Cuenca López. Universidad de Huelva Jesús Estepa Giménez. Universidad de Huelva Rafael Feito Alonso. Universidad Complutense (Madrid)

Francisco José García Gallardo. Universidad de Huelva

Soledad García Gómez. Universidad de Sevilla J. Eduardo García Díaz. Universidad de Sevilla
Fernando Hernández Hernández. Universidad de Barcelona

Salvador Llinares Ciscar. Universidad de Alicante Alfonso Luque Lozano. Universidad de Sevilla Rosa Martín del Pozo. Universidad Complutense (Madrid)

José Martín Toscano. IES Fernando Herrera (Sevilla) Jaume Martínez Bonafé. Universidad de Valencia F. Javier Merchán Iglesias. Universidad de Sevilla Emilia Moreno Sánchez. Universidad de Huelva. Rosario Ortega Ruiz. Universidad de Córdoba Antonio de Pro Bueno. Universidad de Murcia Fco. de Paula Rodríguez Miranda. Universidad de Huelva

Pedro Sáenz-López Buñuel. Universidad de Huelva Antoni Santisteban Fernández. Universidad Autónoma (Barcelona)

Emilio Solís Ramírez. Catedrático de IES. $\mathbf{M}^{\mathbf{a}}$ Victoria Sánchez García. Universidad de Sevilla. Magdalena Suárez Ortega. Universidad de Sevilla

\section{Consejo asesor}

Manuel Área Moreira. Universidad de La Laguna

Jaume Carbonell. Director Cuadernos de Pedagogía. Barcelona

César Coll. Universidad de Barcelona

Christopher Day. Universidad de Nothingham. U.K.

Juan Delval. Universidad Nacional de Educación a Distancia

John Elliott. Universidad de East Anglia. Norwich. U.K.

José Gimeno Sacristán. Universidad de Valencia

André Giordan. Universidad de Paris VII y Ginebra

Francisco Imbernón. Universidad de Barcelona

Ángel Pérez Gómez. Universidad de Málaga

Rafael Porlán Ariza. Universidad de Sevilla

Francesco Tonucci. Instituto de Pedagogía del C.N.R. Roma

Jurjo Torres Santomé. Universidad de A Coruña 

